Revista Digital Año 9. No 12 - Año 2018. -pág. 1-110

ISSN 1853-1393

Resistencia. Chaco. Argentina - 2018

\title{
EL JUEGO COMO RECURSO DIDÁCTICO PARA EL ALUMNADO CON NECESIDADES EDUCATIVAS ESPECIALES
}

THE GAME AS A TEACHING RESOURCE FOR STUDENTS WITH SPECIAL NEEDS

José M. Bautista-Vallejo1- Isaac Garrido Gómez ${ }^{2}$ - Manuel J. Espigares-Pinazo ${ }^{3}$

Fecha de recepción: 11-10-2017

Fecha de aceptación y versión final: 19-12-2017

Resumen: El presente trabajo trata sobre los beneficios de practicar Educación Física para alumnos y alumnas con necesidades educativas especiales, así como la utilización del juego como recurso didáctico para ayudar a estos alumnos y alumnas a mejorar las relaciones con los demás y favorecer su integración en las clases de Educación Física. Por otro lado, se reflexiona sobre la trascendencia de las actividades deportivas para la consolidación de la igualdad y solidaridad entre personas discapacitadas y no discapacitadas. En este trabajo, las actividades deportivas y los juegos están fundamentados en las características de las diferentes necesidades educativas especiales.

Palabras clave: necesidades educativas especiales - beneficios - Educación Física

Abstract: This paper discusses the benefits of practicing Physical Education for students with special educational needs, and the use of the game as a teaching resource to help these students to improve relations with others and promote their integration into classes Physical Education. On the other hand, it will reflect on the importance of sporting activities to consolidate equality and solidarity between disabled and non-disabled. In this work sporting activities and games will be grounded in the characteristics of different special educational needs.

Key words: special educational needs - benefits - Physical Education

\footnotetext{
${ }^{1}$ Dr. Europeo en Psicopedagogía. Profesor de la Universidad de Huelva (España).

${ }^{2}$ Maestro en Educación Primaria. Concejal de Deportes, Turismo, Juventud e Infancia. Ayuntamiento de Villalba del Alcor (Huelva, España). Universidad de Huelva (España)

${ }^{3}$ Dr. en Tecnología Educativa. Profesor en la Universidad Internacional de la Rioja (España).
} 


\section{Introducción}

¿De qué manera debe actuar el profesorado para atender equitativamente al alumnado discapacitado? ¿Por qué es importante la Educación Física para el alumnado con necesidades educativas especiales y qué beneficios ofrece su práctica? ¿Cuáles son los déficits más comunes, atendiendo a la clasificación exclusiva de Del Toro (2013)? ¿Cuáles son los principales obstáculos que se encuentra el alumnado discapacitado en su afán por practicar una Educación Física adaptada a sus necesidades? ¿Qué medidas puede tomar el profesorado para facilitarles la inclusión y la integración social entre los demás?

Son numerosas las preguntas que se pueden realizar sobre un campo de conocimiento desconocido hasta hace pocos años y que, actualmente, cuenta con diversos estudios e investigaciones que pretenden dar respuesta a los interrogantes anteriores. Como consecuencia de la consulta de diferentes artículos que aluden a las necesidades educativas especiales en el área de Educación Física, se puede afirmar que estas interrogaciones obtienen respuesta desde un ámbito teórico y práctico que se analizarán específicamente en los resultados del análisis (Depauw y Goc Karp, citados por Hernández et al., 2011).

Por último, es importante mencionar que, actualmente, existen multitud de alumnos y alumnas con necesidades educativas especiales y se considera de gran importancia la necesidad de conocer cuál es la mejor manera de desarrollar una práctica educativa inclusiva, atendiendo al alumnado, sin realizar discriminaciones por las distintas capacidades que presente, así como la importancia de dominar los diferentes déficits para ofrecer una educación inclusiva mediante las adaptaciones curriculares que precise el alumnado en cada momento.

\section{Metodología}

Para la búsqueda de los diferentes artículos consultados para realizar el estudio sobre "El juego como recurso didáctico en alumnos con necesidades educativas especiales", se han utilizado diferentes bases de datos como son Google Académico o Google Scholar, base de datos CSIC y base de datos (ERIC) ProQuest. Este estudio pretende, de forma preliminar, un acceso introductorio al campo de estudio propuesto. Es por ello que se delimitan estas fuentes de manera inicial, dejando para futuros accesos al campo de estudio otras que pueden seguir aportando información relacionada.

Debido a las necesidades de la investigación, se tomó la decisión de seleccionar estas bases de datos para búsquedas preliminares. Se tuvo en cuenta para ello la siguiente metodología, basada en 4 pasos (López Noguero, 2002):

Identificar preliminarmente los propósitos que los datos podrían cumplir. Responder a la pregunta sobre los datos necesarios para la investigación presente, pero, también, futura, permite verificar en el futuro nuevos hallazgos y realizar análisis adicionales.

Identificar los datos que deben preservarse, para responder a posibles requerimientos específicos sobre qué datos deben preservarse y bajo qué condiciones.

Identificar los datos que deberían preservarse, lo que lleva a preservar datos, unos u otros, si los que se han encontrado son suficientes o no, de suficiente calidad, etc.

Completar la evaluación de los datos. Finalmente, considerando todos los factores analizados, debe tomarse una decisión sobre la retención de los datos para su preservación.

Así, en cada una de estas bases de datos se ha hecho una búsqueda actualizada de artículos que tratasen sobre el tema en cuestión, ajustando los criterios de inclusión desde el año 2010 hasta el año actual. Sin embargo, ha sido inevitable 
consultar una fuente del año 2005, debido a que consideraba necesario analizar el "Manual de Educación Física adaptada al alumnado con discapacidad", el cual contiene ítems sugerentes para el tema que se está estudiando.

Por otro lado, cabe destacar que los artículos y/o libros, en este tipo de búsqueda bibliográfica sistemática, se han podido ordenar por fecha, situándolos desde los más actuales hasta los menos. Igualmente por relevancia, es decir, los artículos y/o libros más trascendentales se localizaban en los comienzos de las páginas investigadas, mientras que aquellos que no contaban con tanta repercusión se ubicaban en las últimas páginas inspeccionadas. La trascendencia y repercusión se basa en el uso de metadatos de esos mismos documentos, es decir, aquellos otros datos que definen y describen esos documentos y que cada vez empiezan a ser más frecuentes en su presentación online.

Asimismo, se incluyeron en la exploración citas y patentes con el fin de obtener información destacada por autores consagrados en lo que se refiere al tema de las diferentes discapacidades en Educación Física.

En cuanto a los criterios de exclusión, se puede señalar que solo se buscaron páginas en español en Google Académico y en la base de datos (ERIC) ProQuest, por distintos motivos. Por un lado, se consideraba que, de esta forma, la búsqueda de artículos y/o libros se ajustaría más a los requisitos deseados, es decir, acceso introductorio al campo en países de habla hispana; y, por otra parte, determinados casos únicos también iban a ser exclidos.

De la misma forma que en el Google Académico, en la base de datos (ERIC) ProQuest también se pueden ordenar los artículos de revistas, informes, libros, etc. por fecha y por relevancia, a diferencia de la base de datos CSIC donde solo se puede elegir la fecha de publicación de los documentos, aunque en otro sentido, en esta base de datos, al realizar una búsqueda, se puede seleccionar si las palabras introducidas deben aparecer en los autores, el título, los descriptores, el resumen, etc. o, en cambio, optar por que aparezca en los campos básicos que significa buscar en el título, el resumen, los descriptores, los identificadores y los topónimos.

En lo que respecta a los descriptores, es importante reseñar que se han utilizado descriptores de la misma naturaleza en las distintas bases de datos manejadas.

\section{Resultados}

A continuación, tras la fase de lectura crítica teniendo en cuenta la validez interna, externa y los errores aleatorios, se realiza un análisis en donde se recapitula sobre las necesidades educativas especiales en el ámbito de la Educación Física, atendiendo a diversos apartados que explican o sintetizan la información encontrada sobre diferentes aspectos como son los objetivos propuestos en el área de la Educación Física para el alumnado con discapacidad, los beneficios que concede la Educación Física al alumnado discapacitado, la clasificación de las necesidades educativas especiales y las barreras que se encuentra el alumnado discapacitado en el momento de su aprendizaje y de la participación en el aula, así como las distintas estrategias que facilitan la inclusión de este alumnado en el desarrollo normalizado de la clase.

En definitiva, se trata de una revisión sobre las necesidades educativas especiales y la educación inclusiva en el ámbito de la educación física, recopilando, examinando y evaluando las informaciones que aparecen publicadas en los diferentes artículos científicos (Unesco, en Cisneros y Olave, 2012).

Por otra parte, es importante añadir que, para la búsqueda y la indagación de artículos científicos, han sido utilizados sistemas de información virtuales a los cuales se puede acceder mediante vía electrónica. Estos sistemas se tratan de bibliotecas virtuales, bases de datos implantadas en webs reales, los directorios y los archivos 
informáticos. No obstante, la exploración de artículos en cualquiera de estas estructuras de información se ajustó a unos criterios de legalidad, pertinencia, honestidad y objetividad (Moreno et al., 2010).

Siguiendo a Day (2005), la preparación y elaboración de un trabajo investigador tiene que ver con la organización de dicho escrito, siendo un procedimiento más pragmático la comunicación de los resultados de esta investigación. Además, la actualización de conocimientos de una persona está vinculada al reconocimiento de diferentes contenidos expuestos en una alta cantidad de artículos, por ello, al redactar cualquier trabajo de investigación se debe reclamar una técnica de comunicación que sea uniforme, lacónica e inteligible. De esta forma, agrupamos los resultados de la manera que sigue.

\section{Actuaciones del profesorado ante las necesidades educativas especiales}

Tras una búsqueda consistente y extensa sobre los diferentes estudios donde se alude a los procedimientos más convenientes a seguir para desarrollar una enseñanza productiva y rentable para el alumnado que posee algún tipo de discapacidad, se puede afirmar que la gran mayoría de autores, que hacen alusión al tema de las necesidades educativas especiales en el ámbito de la Educación Física, mencionan la necesidad de mejorar el sistema de información en los centros para que el profesorado se adapte a los ritmos de aprendizaje de los alumnos y alumnas con discapacidad (Serrano et al., 2013), así como evitar las posibles barreras comunicativas que puedan existir entre el profesorado y el alumnado, debido a que este hecho puede desembocar en un clima de indiferencia e ignorancia entre alumnos y alumnas.

Por otro lado, "el juego es un acto voluntario desinteresado e intrascendente, ajeno a la vida ordinaria, limitado espacio-temporalmente como expresión lúdica que caracteriza a una cultura dentro de unos parámetros de tensión y orden intrínsecos" (Cagigal, citado por Suari, 2005: 26). Más allá de esta definición, cuando se hace referencia a la Educación Física adaptada al alumnado con necesidades educativas especiales, se sugiere que sea el profesor quien realice un programa de actuación en el cual planifique una intervención que ofrezca al alumnado una respuesta ante cualquier necesidad educativa especial. Para ello, resulta indispensable la necesidad de que el profesorado sea consciente del nivel de complejidad y participación que ello requiere.

En concreto, el diseño de juegos debe hacer posible el enriquecimiento de los procesos terapéuticos de tal manera que sean satisfactorias y eficientes para las personas con algún tipo de discapacidad, como la auditiva (Peñeñory, Bacca y Cano, 2018).

Siguiendo a Hernández (2009), el alumnado discapacitado debe seguir el mismo currículo establecido para la etapa educativa en concreto, realizándose las adaptaciones curriculares necesarias para desarrollar la función docente de manera apropiada. Según la autora, estas adaptaciones curriculares favorecen la implicación del alumno o alumna en el desarrollo afectivo y social de la clase, ayuda al desarrollo psicomotor y del lenguaje y fomenta la capacidad de representación y resolución de problemas.

Para lograr un incremento en el reconocimiento del entorno inmediato se precisa de la programación de actividades o juegos enfocados al conocimiento del mismo, permitiéndole al alumnado utilizar los diferentes objetos, de forma libre, para alcanzar una mayor independencia y autonomía. Asimismo, el uso del cuerpo como elemento esencial de comunicación e interacción facilita el desarrollo motor de los alumnos y alumnas. Además, la actividad física adaptada constituye un instrumento de aprendizaje para el desarrollo cognitivo y emocional, posibilitando una integración social por el carácter espontáneo e innato de la Educación Física (Gómez, 2013). 
La Educación Física es una parte principal en los derechos que tienen las personas en cuanto a la educación, siendo necesario adaptar las actividades deportivas y prácticas educativas para atender a todo el alumnado de manera equitativa (Nicoletti y García, citados por Darretxe et al., 2016).

Algunos estudios realizados sobre las actitudes de los profesores hacia el alumnado con discapacidad, presentan dos variables, las cuales estarán relacionadas con el alumnado con necesidades educativas especiales y con el profesorado. Entre aquellas variables relacionadas con el profesorado se puede encontrar la edad, el género y la experiencia previa.

En cuanto a la edad, diversos estudios afirman que los profesores más jóvenes tienen más dificultades para llevar a cabo una educación inclusiva del alumnado con discapacidad que aquellos profesores con más edad (Depauw y Goc Karp, citados por Hernández et al., 2011).

Por otra parte, el género es un aspecto relevante, según algunos autores, en la actitud que muestra el profesorado ante las necesidades educativas especiales. Por lo general se afirma que aquellas personas de género femenino tienen unas conductas más positivas frente al tema de las discapacidades y fomentan de forma más adecuada el aprendizaje de este tipo de alumnado (Aloia et al., Downs y Williams, Schmidt-Gotz y Doll-Tepper, Folsom-Meek y Rizzo, Meegan y MacPhail, citados por Hernández et al., 2011).

Por último, cabe destacar que, según diferentes estudios, el profesorado que cuenta con una mayor experiencia docente tiene mayor predisposición para la enseñanza del alumnado con necesidades educativas especiales que el colectivo de profesores que posean menos experiencia en el ámbito académico (Hernández et al., 2011).

Actualmente, se pueden encontrar ciertas actitudes basadas en un prejuicio sobre las características que presentan algunas personas con necesidades educativas especiales (Martínez y Bilbao citados por Abellán, 2015). La posible evaluación de los pensamientos del alumnado resulta fundamental para la integración social y emocional de los alumnos y alumnas con discapacidad en el desarrollo afectivo-social de la clase de Educación Física (Suriá et al., citados por Abellán, 2015).

En otro sentido, la educación inclusiva se plantea fundamentalmente como un aspecto inherente a la programación del docente, puesto que la enseñanza constituye un campo de acción donde se deben tener en cuenta aquellas peculiaridades extraídas del contexto educativo (Echeita citado por Caus et al., 2013). Sin embargo, el profesorado deberá contar con un instrumento apropiado que le permita programar actividades precisas a fin de transmitir adecuadamente el trabajo planificado al alumnado con discapacidad, no obstante, el profesorado tendrá la responsabilidad de poseer una actitud favorable hacia las necesidades educativas especiales (Caus et al., 2013).

\section{Objetivos del área de Educación Física para el alumnado con discapacidad}

Al hilo de lo expuesto anteriormente, Castillo et al. (2015) afirman que existen personas que tienen diversos problemas a la hora de incorporarse a las situaciones de la vida cotidiana, instalando un clima de dificultad para integrarse socialmente y desenvolverse motrizmente en las diferentes actividades, surgiendo, así, problemas de relación con los demás. Como es observable durante el estudio de casos de alumnos y alumnas con necesidades educativas especiales, existen diversos fines de la Educación Física para este alumnado (López y García, 2018), destacando por encima del resto el empleo y disfrute del juego motriz como oportunidad esencial para integrar a los infantes en una sociedad normalizada.

Sin embargo, se pueden encontrar múltiples objetivos, los cuales variarán según el tipo de alumnado que presente la discapacidad, como mejorar la salud, 
aspecto primordial en alumnos o alumnas con necesidades educativas especiales puesto que podrían presentar alguna limitación motriz; mejorar la autoestima, los juegos deben tener unos objetivos alcanzables por los alumnos o alumnas para que se sientan motivados; desarrollar al alumnado de manera integral, el juego debe ser el inicio del proceso educativo mediante el cual los discentes adquieran otros tipos de aprendizajes; fomentar el desarrollo social, a través del uso del juego cooperativo y colaborativo como recurso didáctico se trabaja la socialización entre iguales; y, desarrollar los valores intrínsecos del deporte, son muchos los valores deportivos que se ponen en juego con la realización y práctica de cualquier actividad física, como son el compañerismo, la colaboración, la tolerancia, etc. (Castillo et al., 2015).

No obstante, resulta vital el conocimiento de las circunstancias distintivas de cada alumno o alumna para adaptar la actividad y, de este modo, permitir al alumnado gozar y juguetear de manera extraordinaria (Chiva-Bartoll y Salvador-García, 2015).

En el área de la Educación Física, el alumnado deberá alcanzar unos objetivos mínimos que se basan en la educación inclusiva adaptada a sus necesidades (Mendoza citado por Abarca, Julián y García, 2013).

\section{Beneficios que aporta la Educación Física al alumnado con necesidades educativas especiales}

Siguiendo a Ríos et al. (2014), la actividad física constituye un elemento fundamental para la mejora de la calidad de vida y de la salud, mediante beneficios fisiológicos, psicológicos y sociales, avalados por varias investigaciones científicas. Los beneficios fisiológicos son el fortalecimiento de los huesos y de los músculos y la reducción del riesgo de padecer enfermedades cardiovasculares (Jaramillo-Alcázar, Luján-Mora y Salvador-Ullauri, 2018).

Por otra parte, los beneficios psicológicos hacen alusión a la mejora del estado de ánimo y la disminución del riesgo de padecer estrés y, por último, el fomento de la sociabilidad y las relaciones interpersonales con los demás se encuadran dentro de los beneficios sociales (Ramírez, Gallardo y Cansino, 2015).

\section{Desarrollo integral del alumnado}

Un aspecto importante de la educación es el dedicado al desarrollo integral. Buena parte de los estudios señalan que los beneficios que otorga la Educación Física al alumnado con necesidades educativas especiales, también son beneficios asignados al alumnado que no padece alguna discapacidad.

Entre estos beneficios aparecen la contribución al desarrollo integral de la persona y otros como el control del sobrepeso y obesidad, una mayor mineralización de los huesos y la disminución de padecer osteoporosis en una vida futura adulta, una mejor maduración del sistema nervioso motor y aumento de las destrezas motrices y un mejor rendimiento escolar y sociabilidad (Cáceres Zúñiga, Granada Azcárraga y Pomés Correa, 2018; Cerisola, 2019).

\section{Barreras que tiene el alumnado con discapacidad para el aprendizaje y la participación}

Según Darretxe et al. (2016), en una gran cantidad de ocasiones las dificultades que se encuentra el alumnado con discapacidad se debe a obstáculos que no están relacionados con el déficit que padece, sino que se corresponden con problemas externos como son la falta de recursos materiales y personales, la organización, los altos ratios de alumnos y alumnas, la falta de formación del profesorado, las actitudes negativas frente a las discapacidades y la falta de accesibilidad al contexto educativo.

No obstante, también se pueden citar dificultades desde la propia integración social del alumnado ya que existen muchos prejuicios y creencias erróneas sobre las 
personas con discapacidad que, en cierta medida, restringe su participación e implicación en las tareas cotidianas (Felipe y Garoz, citados por Darretxe et al., 2016).

Para Serrano et al. (2013), existen demasiadas barreras que dificultan y obstaculizan la implicación de las personas con discapacidad en la ejecución de las actividades referentes a la vida cotidiana.

Han sido numerosos los estudios dedicados a establecer una serie de barreras que se interponen en el camino de las personas con algún tipo de discapacidad en el momento de realizar actividades en la vida cotidiana o en el área educativa (Manghi, Saavedra y Bascuñan, 2018). Se pueden destacar como principales barreras personales y ambientales la falta de motivación, la dependencia de terceros, los materiales inapropiados según las condiciones motrices y la consideración de la actividad física como un riesgo que empeoraría la condición del discapacitado (Buffart citado por Serrano et al., 2013).

\section{Estrategias que ayudan al alumnado discapacitado en su aprendizaje y participación}

Al igual que se encuentran barreras que dificultan el aprendizaje del alumnado con discapacidad, sería importante agregar que se hallan diversas estrategias que posibilitan la inclusión del alumnado con discapacidad en el área de Educación Física, mejorando su aprendizaje y haciéndolos partícipes de su propio proceso de enseñanza. Se pueden clasificar en el fomento de actitudes positivas frente a las discapacidades, el conocimiento de las diferentes limitaciones que tiene el alumnado y la apuesta por unas estrategias metodológicas inclusivas (Darretxe et al., 2016).

Actualmente, se pueden encontrar investigaciones y programaciones educativas en el área de Educación Física que pretenden transformar las actitudes negativas de las personas frente a las necesidades educativas especiales por un clima de tolerancia y respeto (Felipe y Garoz, 2014; Felipe y Garoz, citados por Darretxe et al., 2016).

Asimismo, para alcanzar una educación inclusiva se antoja necesaria la creación de estrategias metodológicas como las distintas adaptaciones curriculares que se pueden realizar en la clase de Educación Física y las diversas formas de agrupar al alumnado para que haya una interacción social e interrelación entre los alumnos y alumnas (Darretxe et al., 2016).

Sin embargo, no se han encontrado muchas investigaciones donde se detallen determinados tipos de metodologías a seguir o estrategias específicas para desarrollar correctamente la función docente en Educación Física con el alumnado que presente algún tipo de discapacidad. Además, se puede atribuir la falta de investigaciones sobre la atención a la diversidad a la insuficiente formación del profesorado de Educación Física, la heterogeneidad del alumnado y la cuantiosa falta de recursos tanto materiales como personales (Mendoza, citado por Abarca, Julián y García, 2013).

Siguiendo a Pérez-Tejero et al. (2012), el deporte no tiene una gran repercusión mediática entre las administraciones, contando con pocas investigaciones sobre las necesidades educativas especiales.

\section{Discusión y conclusiones}

Con el análisis de los resultados obtenidos se procederá a la síntesis de los mismos, evaluando críticamente los diferentes estudios analizados, así como las distintas investigaciones y artículos consultados.

Para comenzar, sería importante destacar que la mayoría de autores que estudian e investigan las actitudes del profesorado de Educación Física con respecto a las necesidades educativas especiales ponen el énfasis en la obligación de mejorar la comunicación entre docentes y discentes, así como el fomento de cursos de formación que ayude al profesorado a tener un mejor conocimiento sobre las distintas discapacidades. Se considera que el papel del profesorado es muy relevante por 
cuanto que éstos pueden manifestar un importante interés por las discapacidades en ámbitos educativos, en cuyo ámbito educativo son los encargados de enseñar al alumnado en un clima de tolerancia, respeto, acomodo y cordialidad.

El hecho de programar actividades o juegos para atender a todo el alumnado equitativamente depende del profesorado. Por ello, resulta indispensable tener conciencia sobre las posibles o las presentes necesidades educativas especiales para desarrollar la función docente atendiendo al alumnado según sus posibilidades y exigencias, debido a los derechos educativos de todo miembro de la comunidad educativa (Nicoletti y García, 2015).

Por lo que se refiere al ámbito emocional del alumnado discapacitado, Hernández (2009) y Gómez (2013) coinciden en la relevancia del juego para favorecer el desarrollo afectivo-social de este tipo de alumnos y alumnas, puesto que las actividades cooperativas y/o grupales constituyen una importante fuente de comunicación e interrelación entre ellos, ayudándoles de manera natural por la idiosincrasia del área de Educación Física.

En otro sentido, Hernández et al. (2011) reconocen la existencia de diversas variables que afectan directa o indirectamente al comportamiento del profesorado frente a las necesidades educativas especiales. El aspecto relacionado con la edad del profesorado, cuyos distintos estudios afirman que al profesorado más joven les entraña más dificultad desarrollar una educación inclusiva, resulta bastante llamativo puesto que la concepción de educación inclusiva debería de ir cambiando con el paso de los años, siendo el profesorado joven quien impulse a las personas con discapacidad hacia la práctica de una educación basada en la integración social con independencia de las capacidades de cada alumno o alumna.

Tal vez, según los diversos estudios que Hernández et al. (2011) citan en su artículo, sea necesario reemplazar los prejuicios del alumnado sobre las personas con discapacidad para favorecer las relaciones positivas entre ellos, consiguiendo una práctica normalizada de Educación Física dentro de unos parámetros de socialización entre el alumnado.

En lo que se refiere a la organización de la propuesta curricular, cabe destacar que los objetivos que se planteen en el área de Educación Física para el alumnado deben ser concisos y fácilmente alcanzables. Haciendo referencia a lo expuesto por Chiva-Bartoll y Salvador-García (2015), se discrepa en la propuesta de objetivos a conseguir por el alumnado discapacitado, puesto que se entiende que mejorar la salud, mejorar la autoestima, desarrollar al alumnado de manera integral y desarrollar los valores intrínsecos del deporte son objetivos que el alumnado no discapacitado debe alcanzar con el planteamiento de las diferentes sesiones de Educación Física. Se considera que estos objetivos son generales para todo el alumnado, no existiendo ningún objetivo que aluda específicamente al alumnado con necesidades educativas cuyo origen esté relacionado con la diversidad funcional o la discapacidad intelectual.

Dada la amplitud con que se presentan las diversas formas de atención a la diversidad en centros educativos, se precisarán una serie de medidas a modo de prácticas educativas en donde, además de las adaptaciones curriculares individualizadas, se desarrollarán otra serie de medidas en donde los distintos formatos que alcanzan los agrupamientos, el fomento del trabajo en grupo, la combinación de actividades, etc., serán de vital importancia (González, Alba y Mesa, 2018). Por ello, coincidimos con Mendoza (citado por Abarca, Julián y García, 2013) en que el área de Educación Física debe desarrollar una educación inclusiva atendiendo a las exigencias del alumnado, centrado en su persona.

Estas medidas reportan unos beneficios al alumnado que presenta, por ejemplo diversidad funcional o intelectual, puesto que se adecuan a las distintas capacidades de alumnos y alumnas. Se entiende que la práctica de actividades físicas constituye la mejor manera de desarrollar al alumnado psicológica y físicamente, además de 
ofrecerle al alumnado la posibilidad de mejorar las relaciones con los demás, debido a la naturaleza socializadora de esta área educativa (Núñez, Aravena, Oyarzún, Tapia y Salazar, 2018).

Mediante la Educación Física los alumnos y alumnas aprenden a valorar a los demás y a aceptarlos con sus defectos y virtudes, interpretando las capacidades de cada persona de forma positiva. Asimismo, esta área contribuye al desarrollo integral del alumnado a través de contenidos imprescindibles como la solidaridad, el respeto, la tolerancia, la responsabilidad, la diversión, la igualdad y la libertad.

En el abordaje de una extensa variedad de formas de discapacidad, y cada vez más frecuente en algunos ambientes por el principio de atención personalizada, una frecuente manifestación en la práctica educativa acorde a las necesidades del alumnado se concreta en las adaptaciones curriculares para las personas que presenten algún tipo de discapacidad (Chiva-Bartoll y Salvador-García, 2015).

No obstante esto, no todos los déficits necesitarán la misma. Aquel alumnado con déficits en el ámbito cognitivo, requerirá una enseñanza más pausada para ayudarles a realizar las actividades, respetando su ritmo de aprendizaje (Serrano et al., 2013). En relación con los déficits motóricos, las actividades deberán estar adaptadas de manera que el alumnado tenga la ocasión de manipular y juguetear con los materiales acondicionados para la práctica de Educación Física. Por último, el alumnado discapacitado sensorialmente necesitará de una atención personalizada para aumentar y fomentar la comunicación y el trabajo colaborativo entre los alumnos y alumnas.

Ahora bien, aún realizando adaptaciones curriculares, se pueden encontrar dificultades propias de este alumnado relacionadas con la inseguridad, la debilidad y la delicadeza, aparte de los problemas generales que se encuentra el alumnado discapacitado en el área de Educación Física y que Serrano et al. (2013) y Felipe y Garoz (2014) citan en sus respectivos artículos.

Los estudios manifiestan que la mayoría del alumnado discapacitado se siente inseguro y débil frente a la realización de cualquier actividad. Más allá de la falta de confianza, autoconcepto y asertividad que este alumnado haya podido autogenerar a lo largo de su vida, autores como Caus et al. (2013) ponen de manifiesto la importancia de los climas de confianza que el profesorado debe construir a lo largo del proceso y jornada, ecosistema que invite a minimizar la falta de confianza y a generar un ambiente propicio para la realización de las actividades y, consiguientemente, el logro de los objetivos de esta área.

En este momento, el profesorado debe tener la capacidad de transformar el pensamiento negativo de este alumnado para que se valoren y se vean útiles en una sociedad abierta, tolerante y racional. A través del fomento de actitudes positivas, el alumnado discapacitado podrá sentirse valorado, desapareciendo de forma gradual las inseguridades con las que afronta las diferentes actividades. Además, la conciencia sobre las distintas necesidades educativas especiales ayudará al profesorado a tomar las mejores decisiones en el desempeño de sus funciones docentes.

tras el análisis crítico de las diferentes fuentes consultadas se puede afirmar que la mejor manera de atender al alumnado discapacitado es mediante las distintas adaptaciones curriculares que se hagan al programar las actividades, así como la integración del alumnado en la dinámica general de la clase, posibilitando la práctica de Educación Física a través de juegos cooperativos y colaborativos mediante los cuales el alumnado con necesidades educativas especiales participe en el desarrollo afectivo-social de la clase.

En definitiva, tras el análisis crítico de las diferentes fuentes consultadas se puede afirmar que las necesidades educativas especiales abarcan un gran campo de estudio y conocimiento, precisando del interés y del análisis de los autores por definir 
las pautas más apropiadas para atender a todo el alumnado discapacitado de manera ecuánime, en este caso desde el campo de la Educación Física.

Por último, la educación inclusiva de este tipo de alumnado no depende solo del desempeño docente y de toda iniciativa del mismo, sino que se encuentran diversas variables que interfieren en el proceso de enseñanza-aprendizaje como pueden ser el contexto educativo, la situación demográfica del centro y la educación que reciben los alumnos y alumnas de sus progenitores, entre otros.

\section{Referencias Bibliográficas}

Abarca, A., Julián, J. A. y García, L. (2013). Adaptación del currículum ordinario de Educación Física en Educación Primaria y propuesta metodológica para alumnado escolarizado en centros de Educación Especial. Recuperado de https://dialnet.unirioja.es/servlet/articulo?codigo $=4530404$

Abellán, J. (2015). Actitudes hacia la discapacidad de los futuros maestros de Educación Física. Sportis: Revista Técnico-Científica del Deporte Escolar, Educación Física y Psicomotricidad. Recuperado de http://revistas.udc.es/index.php/SPORTIS/article/view/1414/pdf_9

Cáceres Zúñiga, F., Granada Azcárraga, M., y Pomés Correa, M. (2018). Inclusión y Juego en la Infancia Temprana. Revista latinoamericana de educación inclusiva, 12(1), 181-198.

Castillo, F., Andrade, S., Arias, N., Cabrales, F. y Díaz, J. (2015). Necesidades educativas especiales y Educación Física: Las actividades en la naturaleza como instrumento socializador. Revista do Programa de Pós Graduaçao Interdisciplinar em $\begin{array}{llll}\text { Estudos do Lazer/UFMG. Recuperado } & \end{array}$ https://seer.ufmg.br/index.php/licere/article/view/1265

Caus, N., Santos, E., Blasco, J. E., Vega, L., Mengual, S. y Yangüez, E. (2013). Procedimiento de actuación ante la inclusión de alumnado con discapacidad en el área de educación física (PAIADEF). Apunts: Educación Física y Deportes. Recuperado de http://www.revistaapunts.com/es/hemeroteca?article $=1589$

Cerisola, A. (2019). El poder del juego: el rol del pediatra para promover el desarrollo en niños pequeños. Archivos de Pediatría del Uruguay, 90(1), 25-28.

Chiva-Bartoll, O. y Salvador-García, C. (2015). Juegos motrices para niños y niñas con parálisis cerebral. Revista de Transmisión del Conocimiento Educativo y de la Salud, 7(2), 221-246. Recuperado de http://www.trances.es/papers/TCS\%2007_2_5.pdf

Cisneros, M. (2008). Ciencia y lenguaje en el contexto académico. Revista Lenguaje, $36(1)$, 117-137.

Recuperado de http://media.utp.edu.co/referenciasbibliograficas/uploads/referencias/articulo/634ciencia-y-lenguaje-en-el-contextoacademicopdf-RO72h-articulo.pdf

Cisneros, M. y Olave, G. (2012). Redacción y publicación de artículos científicos: enfoque discursivo. Bogotá, Colombia: Ecoe Ediciones. Recuperado de https://discurso.files.wordpress.com/2012/02/articulos-cientificos.pdf

Darretxe, L., Gaintza, Z. y Etxaniz, J. (2016). Hacia una educación más inclusiva del alumnado con discapacidad en el área de Educación Física. EmásF, Revista Digital de Educación Física, 7(41), 10-20. Recuperado de http://emasf.webcindario.com/Hacia_una_educacion_mas_inclusiva_del_alumnado_co n_discapacidad_en_el_area_de_EF.pdf

Day, R. (2005). Cómo escribir y publicar trabajos científicos. Washington: Organización Panamericana de la Salud. Recuperado de http://www.bvs.hn/Honduras/pdf/Comoescribirypublicar.pdf

Del Toro, V. (2013, 1 junio). El juego como herramienta educativa del Educador Social en actividades de animación sociocultural y de ocio y tiempo libre con niños con 
discapacidad. Revista de Educación Social. (16), 1-13. Recuperado de http://www.eduso.net/res/pdf/16/jue_res_16.pdf

Felipe, C., y Garoz, I. (2014). Actividad físico-deportiva en programas de cambio de actitudes hacia la discapacidad en edad escolar: Una revisión de la literatura. Cultura, Ciencia y Deporte: Revista De Ciencias De La Actividad Física y Del Deporte De La Universidad Católica De San Antonio. Recuperado de http://ccd.ucam.edu/index.php/revista/article/view/462/305

Gómez, O. (2013, 2 junio). La motricidad en TEA-AF y SA: Propuesta para la mejora del déficit motor y de la interacción social. Recuperado de https://uvadoc.uva.es/bitstream/10324/3817/1/TFM-G\%20199.pdf

González, P. C., Alba, B. G. y Mesa, M. D. F. S. (2018). Agrupamientos escolares y retos para la educación inclusiva en infantil y primaria. Tendencias pedagógicas, (32), 75-90.

Heredia, J., y Duran, D. (2013). Aprendizaje cooperativo en Educación Física para la inclusión de alumnado con rasgos autistas. Revista nacional e internacional de educación inclusiva. 6(3), 25-40. Recuperado de http://grupsderecerca.uab.cat/grai/sites/grupsderecerca.uab.cat.grai/files/INV\%20HER E DIA.pdf

Hernández, B. (2009). El desarrollo motor y perceptivo del niño discapacitado. Revista Digital, 13(130), 1-15. Recuperado de http://www.discapacidadonline.com/wpcontent/uploads/Discapacidad-infantil-ydesarrollo-perceptivo-motor.pdf

Hernández, F. J., Casamort, J., Bofill, A. M., Niort, J., y Blázquez, D. (2011). Las actitudes del profesorado de Educación Física hacia la inclusión educativa: Revisión. Apunts: Educación Física y Deportes. Recuperado de http://www.revistaapunts.com/es/hemeroteca?article=1462

Jaramillo-Alcázar, A., Luján-Mora, S. y Salvador-Ullauri, L. (2018). Educación Inclusiva: Juegos Serios Móviles para Personas con Discapacidades Cognitivas. Enfoque UTE, 9(1), 53-66.

López, I. G., y García, D. M. (2018). La formación permanente como herramienta para mejorar la intervención del maestro de educación física con alumnado con discapacidad. Retos: nuevas tendencias en educación física, deporte y recreación, (33), 118-122.

López Noguero, F. (2002). El análisis de contenido como método de investigación. XXI, Revista de Educación, 4, 167-179.

Manghi, D., Saavedra, C., y Bascuñan, N. (2018). Prácticas Educativas en Contextos de Educación Pública, Inclusión Más Allá de las Contradicciones. Revista latinoamericana de educación inclusiva, 12(2), 21-39.

Martínez, M.A. y Bilbao, M.C. (2011). Los docentes de la universidad de Burgos y su actitud hacia las personas con discapacidad. Revista Española sobre la Discapacidad intelectual Siglo Cero, 42 (4), 50-78. Recuperado de http://sid.usal.es/idocs/F8/ART19441/240-8\%20Mart\%C3\%ADnez.pdf

Moreno, F., Marthe, N. y Rebolledo, L. (2010). Cómo escribir textos académicos según normas internacionales. Barranquilla, Colombia: Editorial Universidad de Norte. Recuperado de https://unlugarpropio.files.wordpress.com/2013/08/cc3b3mo-escribirtextosacadc3a9micos.pdf

Nicoletti, J. A., y García, G. (2015). El derecho humano a la educación física adaptada. EmásF, Revista Digital de Educación Física, 6(35), 70-78. Recuperado de https://dialnet.unirioja.es/descarga/articulo/5391128.pdf

Núñez, P. C., Aravena, O. A., Oyarzún, J. C., Tapia, J. L. y Salazar, C. M. (2018). Prácticas pedagógicas que favorecen u obstaculizan la inclusión educativa en 
el aula de educación física desde la perspectiva del alumnado y profesorado. Retos: nuevas tendencias en educación física, deporte y recreación, (34), 212-217.

Peñeñory, V. M.; Bacca, A. F. y Cano, S. P. (2018). Propuesta metodológica para el diseño de juegos serios para la rehabilitación psicomotriz de niños con discapacidad auditiva. Campus Virtuales, 7(2), 47-54.

Pérez-Tejero, J., Soto, J., Ocete, C., Alonso, J., García-Hernández, J. J., Blanco, J. A., Coterón, J., Irureta-Goyena, P. y Sampedro, J. (2012). El centro de estudios sobre deporte inclusivo (CEDI): Investigación aplicada, formación y promoción deportiva para personas con discapacidad en acción. Revista Andaluza de Documentación sobre el Deporte. (4), 1-7. Recuperado de http://oa.upm.es/16686/1/INVE_MEM_2012_135899.pdf

Ramírez, G., Gallardo, T. y Cansino, N. (2015). Percepción de los profesores de Educación Física sobre la actividad física en los alumnos con necesidades educativas especiales. Colegio San Jorge, Talca, Chile. Recuperado de http://colegiosanjorgetalca.cl/doc/Percepcion\%20respecto\%20a\%20las\%20NEE\%20de $\% 2010$ \%20profesores\%20de\%20educacion\%20Fisica.pdf

Ramos, M., Ramos, M. y Romero, E. (2003). Cómo escribir un artículo de revisión. Revista de Posgrado de la Vla. Cátedra de Medicina, 4(126), 1-3. Recuperado de http://med.unne.edu.ar/revista/revista126/como_esc_articulo.htm

Ríos, M. (2005). Manual de Educación Física adaptada al alumnado con discapacidad. 2 $2^{\text {a }}$ Edición. Barcelona: Paidotribo.

Ríos, M., Arráez, J. M., Bazalo, P., Enciso, M., Hueli, J. M., Jiménez, E., Cofre, A., Martínez, J. O., Perales, T., Pérez, A., Pérez, J., Rovira-Beleta, E., Sanz, D. y Solís, M. (2014). Plan integral para la actividad física y el deporte. Recuperado de http://femede.es/documentos/Discapacidadv1.pdf

Serrano, C. P., Ramírez, C., Abril, J. P., Ramón, L. V., Guerra, L. Y., y Clavijo, N. (2013). Barreras contextuales para la participación de las personas con discapacidad física. Revista Salud Uis, 45(1), 41-51. Recuperado de http://revistas.uis.edu.co/index.php/revistasaluduis/article/view/3299/3499

Suriá, R., Bueno, A. y Rosser, A. (2011). Prejuicios entre los estudiantes hacia las personas con discapacidad: reflexiones a partir del caso de la Universidad de Alicante. Alternativas: cuadernos de trabajo social, 18, 75-90. Recuperado de https://rua.ua.es/dspace/bitstream/10045/18711/1/Alternativas_18_04.pdf

Tierra, J., Castillo, J. (2009). Educación Física en alumnos con necesidades educativas especiales. Revista Wanceulen E.F. Digital. (5), 52-67. Recuperado de http://rabida.uhu.es/dspace/bitstream/handle/10272/3313/b15548788.pdf 